\title{
Rapid crystallization of glycine using metal-assisted and microwave-accelerated evaporative crystallization: the effect of engineered surfaces and sample volume
}

\author{
Tsehai A.J. Grell, Melissa A. Pinard', Danielle Pettis and Kadir Aslan* \\ Morgan State University, Department of Chemistry, 1700 East Cold Spring Lane, Baltimore, MD 21251, USA \\ ${ }^{1}$ Current Address: University of Florida, Department of Biochemistry and Molecular Biology, 1600 SW Archer Road, Gainesville, FL 32610, USA \\ * Corresponding author: Kadir.Aslan@morgan.edu
}

Abstract

Metal-Assisted and Microwave-Accelerated Evaporative Crystallization (MA-MAEC), is a new approach to crystallization of drug compounds, amino acids, DNA and proteins. In this work, we report our additional findings on the effect of engineered surfaces and sample volume on the rapid crystallization of glycine. With the use of hydrophilic functionalized surfaces and the MA-MAEC technique, glycine crystals $\sim 1 \mathrm{~mm}$ in size were grown in 35 seconds with $100 \%$ selectivity for the $\alpha$-form. The use of moderately hydrophobic surfaces resulted in the growth of glycine crystals only at room temperature. An increase in volume of initial glycine solution $(5-100 \mu \mathrm{L})$ resulted in an increase in crystal size without a significant increase in total crystallization time. Raman spectroscopy and powder X-ray diffraction results demonstrated that the glycine crystals grown on engineered surfaces were structurally identical to those grown using conventional evaporative crystallization.

Keywords: Crystallization, amino acids, glycine, silver island films, plasmonic nanostructures, metal-assisted and microwave-accelerated evaporative crystallization

Citation: T.A.J. Grell, et al. Rapid crystallization of glycine using metal-assisted and microwave-accelerated evaporative crystallization: the effect of engineered surfaces and sample volume. Nano Biomed. Eng. 2012, 4(3), 125-131.

\section{Introduction}

Crystallization of amino acids has been the subject of numerous reports, where new techniques and platforms including polarized laser light irradiation in solution [1], and engineered surfaces such as nanoscale cylindrical pores [2], platforms for micro-droplet solvent evaporation and self-assembled monolayers (SAMs) of alkane thiols on patterned surfaces [3-5] through solvent evaporation, just to name a few, were described. Most notably, Trout et. al. investigated of the role of surface chemistry and morphology of porous polymer surfaces on heterogeneous nucleation of aspirin [6]. The effect of functional groups, such as, carboxyl, hydroxyl, tertiary amide and amine and phenyl ring was found out to have a profound effect on the nucleation and growth of aspirin crystals [6]. These studies demonstrated that the use of engineered surfaces clearly plays an important role in crystal nucleation and growth. However, the crystallization of amino acids and small molecules by conventional evaporative crystallization still takes several hours or longer to complete and require the use of complex platforms. In this regard, there is still a need for new or improved crystallization techniques and platforms, which afford for rapid and selective crystallization of materials of interest.

Recently, Aslan Research Group has developed a new crystallization technique, called Metal-Assisted and Microwave-Accelerated Evaporative Crystallization (MA-MAEC) [7], which is based on the combined use of metal nanostructures deposited onto glass slides via facile chemical route and low power microwave heating. In the MA-MAEC technique, the accelerated crystallization is attributed to the thermal gradient created by selective microwave heating of the sample solution on the metal nanostructures but the not the metal nanostructures themselves. The thermal gradient creates a driving force for the mass transfer of molecules in solution towards the metal nanostructures that act as heterogeneous nucleation sites; hence the technique includes the name of metal- 
assisted crystallization. In our initial publication [7], the proof-of-principle of the MA-MAEC technique was demonstrated using a model amino acid, i.e. glycine. Since then, the MA-MAEC technique has been applied to other amino acids; L-arginine acetate [8] and L-alanine [9] and drug molecules; acetaminophen [10]. We envision that the MA-MAEC technique can be applied to the crystallization of biological macromolecules (i.e., DNA, proteins, etc.). Currently, high-throughput technologies have been employed for the crystallization of biological macromolecules, where more experiments can be carried out with small volume of samples to determine the optimum crystallization conditions. However, crystallization of biological macromolecules still takes a very long time (in the order of days or weeks). In this regard, there is still a need for a technique that affords for better control over the crystallization process, in terms of producing high-quality crystals of peptides and proteins in a significantly shorter time scale (in minutes to a few hours for rapid understanding of the structure-property relationships of protein-based materials. In this regard, the knowledge obtained in our current efforts to crystallize small biological molecules will be utilized in our future efforts the crystallization of biological macromolecules.

In this work,we investigated the effect of engineered surfaces and sample volume on rapid crystallization of glycine using the MA-MAEC technique. In this regard, the heterogeneous nucleation sites (i.e., silver island films, SIFs) for the glycine crystals were enginee ed via a surface modification procedure that includes the attachment of Self-Assembled Monolayers (SAMs) of alkane thiols with - $\mathrm{COOH}$ (hydrophilic) and $-\mathrm{CH}_{3}$ (moderately hydrophobic) and an organic compound with $-\mathrm{NH}_{2}$ (moderately hydrophilic) functionality onto SIFs. Our findings indicate that the use of hydrophilic surfaces in MA-MAEC based crystallization resulted in the rapid $(\sim 35 \mathrm{sec})$ crystallization of glycine and the increase in the volume $(5-100 \mu \mathrm{L})$ of initial glycine solution had no significant effect on the total crystallization time. Characterization of the glycine crystals was carried out via Raman spectroscopy and powder X-ray diffraction (XRD) measurements, which demonstrated that glycine crystals formed on surface engineered SIFs using MAMAEC technique and room temperature evaporation, are identical in nature.

\section{Methods and Materials}

Materials. Sodium hydroxide, ammonium hydroxide, hydrochloric acid $(\mathrm{HCl})$, D-glucose, 1-undecanethiol (1UDET) (98\%), 11- mercaptoundecanoic acid (11-MUDA) (95\%), hexamethylinediamine (HMA) (98\%), Ethanol (200-proof), and glycine (99.5\%) were obtained from Sigma Aldrich. Ethanol (195-proof) was obtained from Pharmco Products Inc. All chemicals were used without further modification.

2.1 Methods. Preparation of SIFs. SIFs were deposited onto blank glass microscope slides by allowing them to soak in a heated silver nitrate/D-glucose solution as 126 previously described [11].

2.2 Preparation of Solutions. A 3.2 M aqueous solution of glycine was prepared by dissolving an appropriate amount of glycine solution slowly in water. Stirring and slight heating $\left(30^{\circ} \mathrm{C}\right)$ were applied to aid the preparation of this concentrated solution. The solution was then allowed to equilibrate to room temperature and then separated into three aliquots. The $\mathrm{pH}$ of the glycine solution was adjusted using $1 \mathrm{M} \mathrm{NaOH}$ and $1 \mathrm{M} \mathrm{HCl}$ to $\mathrm{pH} \mathrm{4,} 6$ and 9. $0.5 \mathrm{mM}$ solutions of 11-MUDA, and HMA were prepared by dissolving the appropriate amounts of solid in 200-proof ethanol. A solution of $0.5 \mathrm{mM}$ of 1-UDET was prepared by pipetting the appropriate quantity of UDET into 200-proof ethanol and mixing until the solution was completely mixed.

\subsection{Preparation of Chemically Engineered Surfaces.} In order to investigate the effect of chemically engineered SIFs, SIFs were soaked in about $25 \mathrm{~mL}$ of $0.5 \mathrm{mM}$ solution of 11-MUDA, HMA and 1-UDETseparately, for $3 \mathrm{hrs}$. These slides, except for UDET-modified SIFs, were then removed for the respective solution and washed thoroughly using ethanol (195-proof)-deionized waterethanol washing sequence to remove any unattached molecules. (All ethanol used for washing was 195-proof). For UDET-modified SIFs washing with deionized water stripped the monolayer and SIFs from the slide, subsequently, multiple ethanol washes were done to ensure this surfaces stayed intact.

2.4 Glycine Crystallization. In the experiments for the investigation of the effect of engineered surfaces on the rapid crystallization of glycine, the following procedure was carried out: a fixed volume $(20 \mu \mathrm{L})$ of freshly prepared glycine solution $(\mathrm{pH} 4,6$ or 9$)$ at $30{ }^{\circ} \mathrm{C}$ was pipetted onto blank glass slide (control experiment) or blank SIFs or surface engineered SIFs and were either heated in a conventional microwave oven (Frigidaire, Model No: FCM09Z03KB, 0.9 cu.ft, $900 \mathrm{~W}$ output power, $100 \%$ power level) or incubated at room temperature (24 $\pm 1{ }^{\circ} \mathrm{C}$, control experiment). The total time taken for the solution to completely evaporate was recorded.

Glycine crystals were characterized by optical microscopy, Raman spectroscopy (iRaman, BW Tek, Inc, USA) and powder XRD. XRD data for glycine crystals placed in a capillary tube with thin walls $(0.02 \mathrm{~mm})$ were collected using an in-house X-ray generator (MicroMax 7, Rigaku/MSC, The Woodlands, TX) and a Raxis4 ${ }^{++}$image plate detector (Rigaku/MSC), which is housed at the Core Facilities of the Department of Pharmaceutical Sciences, University of Maryland School of Pharmacy. The distance between the detector and samples were kept constant at $75 \mathrm{~mm}$. The radiation source was $\mathrm{CuK}$ (wavelength: 0.54 $\mathrm{nm}$ ). The 2-D XRD data was collected at $0^{\circ} \leq \delta \leq 120^{\circ}$ at values of $0^{\circ} \leq 2 \theta \leq 40^{\circ}$.

The contact angles for the three glycine solutions $(\mathrm{pH}$ 
4, 6 and 9) on the three engineered surfaces that contain SAMs of HMA, 11- MUDA and 1-UDET were measured using a Kruss Drop Shape Analysis System (DSA) Model Number $10 \mathrm{Mk} 2$.

\subsection{Effect of Sample volume.}

To investigate the effect of sample volume on crystallization time, four different volumes, 5, 20, 10, 50 and $100 \mu \mathrm{L}$ of glycine solutions were pipetted onto HMA-, MUDA- and UDET-modified SIFs. The time taken for the complete evaporation of the solvent, during microwave heating and incubation at room temperature, was recorded. In eight control samples, the crystallization of glycine of sample volumes 5, 20, 10, 50 and $100 \mu \mathrm{L}$ were carried out on blank glass slides. Half of the samples were microwave heated and the other half were incubated at room temperature. For the glycine solution volumes of 50 and $100 \mu \mathrm{L}$, silicon wells were placed on the slides to prevent the glycine solution from spilling off surfaces.

\section{Results and Discussions}

Inour initial previous publication [7], we demonstrated the proof-of-principle of our new crystallization technique, MA-MAEC, using glycine, blank glass slides and unmodified SIFs. In the current work, we furthered our understanding of crystallization of glycine by studying the effect of different functional groups on SIFs, $\mathrm{pH}$ and volume of the initial solution. It is necessary to comment on the chemical nature of glycine for the sake of better understanding of the use of MA-MAEC based crystallization of glycine. Glycine is the simplest amino acid containing one carboxylic and amine groups and thus is frequently employed as a model amino acid in the demonstration of new crystallization techniques and tools. Glycine, like all amino acids, form peptide chains by a condensation reaction between and amine group and carboxylic group on two different amino acids to via an amide bond. Based on the $\mathrm{pH}$ of the glycine solution, these groups could be protonated or deprotonated, allowing electrostatic interactions to occur and different forms of glycine crystals are formed [3]. Taking the chemical nature of glycine (i.e., the presence of carboxylic and amine groups) into account, three functional groups, $-\mathrm{COOH},-\mathrm{NH}_{2}$ and $-\mathrm{CH}_{3}$ were introduced to the SIFs using SAMs of MUDA, HMA and UDET respectively (Scheme 1). The assembly of glycine on each of these engineered surfaces is thought to be different:

i) The assembly of monolayer of glycine on MUDAmodified SIFs, which presents functional - $\mathrm{COOH}$ groups is expected to take place via electrostatic interactions between the $\mathrm{NH}_{2}$ groups of the glycine molecule and $-\mathrm{COO}^{-}$(depending on the $\mathrm{pH}$ of glycine solution) groups on SIFs.

ii) The assembly of monolayer of glycine onto HMAmodified SIFs occurs via the interactions between the $-\mathrm{COO}^{-}$groups of the glycine molecule and $-\mathrm{NH}_{2}$ groups on SIFs.

http://nanobe.org

iii) The assembly of initial glycine molecules onto UDET-modified SIFs is due to hydrophobic interactions between $-\mathrm{CH}_{2} / \mathrm{CH}_{3}$ groups.

Subsequent glycine molecules assemble onto the monolayer of glycine molecules via electrostatic interactions between both the carboxylic and amine groups of glycine on all surface engineered SIFs. The functional groups on SIFs are thought to lower the interfacial energy between SIFs and glycine crystals, which promotes the heterogeneous nucleation of glycine crystals on SIFs. The presence of $-\mathrm{COOH},-\mathrm{NH}_{2}$ and $-\mathrm{CH}_{3}$ groups on SIFs results in hydrophilic, moderately hydrophilic and moderately hydrophobic surfaces, respectively. It is important to characterize these surfaces to confirm that the surface modification process was carried out as intended. The hydrophilic/hydrophobic nature of the engineered surfaces was assessed by measuring the contact angle of a drop of $3.2 \mathrm{M}$ glycine solution at $\mathrm{pH}$ 4, 6 and 9, and was shown in Table 1.The contact angle for all glycine solutions on a blank glass slide and SIFs were less than $10^{\circ}$ (i.e., hydrophilic surface) and $26-29^{\circ}$ (moderately hydrophilic). Surface modification of SIFs with MUDA results in the presence of $-\mathrm{COOH}$ terminal functional groups (as depicted in Scheme 1), which are known to create a hydrophilic surface. The contact angle of glycine solutions on MUDA-modified SIFs is similar to that of blank glass (less than $10^{\circ}$ ) at $\mathrm{pH} 4$ and increases to $14^{\circ}$ at $\mathrm{pH} 9$, which proves the conversion of moderately hydrophilic SIFs into hydrophilic surfaces. On HMAmodified SIFs, the contact angle of glycine solutions is between $38^{\circ}-48^{\circ}$, which implies a moderately hydrophilic surface due to the presence of $-\mathrm{NH}_{3}{ }^{+}$groups on SIFs. The attachment of UDET onto SIFs, which results in the presence of $-\mathrm{CH}_{3}$ terminal functional groups on SIFs, further increases the contact angle of glycine solutions up to $58^{\circ}$ (moderately hydrophobic).

Subsequently, as shown in images placed in Table 1, initial glycine solution spreads over a larger area on blank glass slides and MUDA-modified SIFs (due to

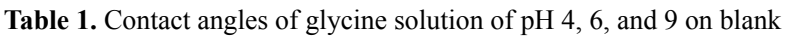
glass slides and surface engineered SIFs. The insets show the digital image of the drop of glycine on the respective surfaces. The contact angles for SIFs were 26.0, 28.5, 29.0, respectively.

\begin{tabular}{|c|c|c|c|c|c|c|}
\hline & \multicolumn{6}{|c|}{ Contact angle (glycine drop) } \\
\hline $\begin{array}{c}\mathrm{pH} \\
\text { Glycine }\end{array}$ & & 4 & & 6 & & 9 \\
\hline $\begin{array}{l}\text { Glass } \\
\text { (Blank) }\end{array}$ & $<10^{\circ}$ & & $<10^{\circ}$ & & $<10^{\circ}$ & \\
\hline HMA & $38^{\circ}$ & & $43^{\circ}$ & & $48^{\circ}$ & $\infty$ \\
\hline MUDA & $<10^{\circ}$ & & $13^{\circ}$ & & $15^{\circ}$ & \\
\hline UDET & $47^{\circ}$ & 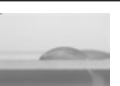 & $53^{\circ}$ & $\omega^{2}$ & $58^{\circ}$ & 2 \\
\hline
\end{tabular}


Table 2. Summary of results for the crystallization of glycine from 20 $\mathrm{ml}$ initial solution using MA-MAEC technique and control experiments.

\begin{tabular}{|c|c|c|c|c|c|c|}
\hline & \multicolumn{2}{|c|}{$\begin{array}{c}\text { Microwave Heating } \\
\text { Crystal morphology } \\
\text { Time } \\
\text { (3.2 M Glycine) }\end{array}$} & \multicolumn{3}{c|}{$\begin{array}{c}\text { Room Temperature } \\
\text { Crystal morphology } \\
\text { Time } \\
(3.2 \text { M Glycine })\end{array}$} \\
\hline pH & HMA & MUDA & UDET & HMA & MUDA & UDET \\
\hline & N/A\# & - & - & $\alpha$ & $\alpha: \gamma$ & $\alpha: \gamma$ \\
$\mathbf{4}$ & $30 \pm 6 \mathrm{sec}$ & $26 \pm 3 \mathrm{sec}$ & $132 \pm 17 \mathrm{sec}$ & $15 \pm 1 \mathrm{~min}$ & $21 \pm 2 \mathrm{~min}$ & $38 \pm 5 \mathrm{~min}$ \\
\hline & $\alpha$ & $\alpha$ & - & $\alpha: \gamma$ & $\alpha$ & $\alpha: \gamma: \beta$ \\
$\mathbf{6}$ & $45 \pm 1 \mathrm{sec}$ & $35 \pm 3 \mathrm{sec}$ & $130 \pm 17 \mathrm{sec}$ & $26 \pm 1 \mathrm{~min}$ & $17 \pm 2 \mathrm{~min}$ & $34 \pm 0 \mathrm{~min}$ \\
\hline & $\alpha: \gamma$ & - & - & $\alpha: \gamma$ & $\alpha: \gamma$ & - \\
$\mathbf{9}$ & $68 \pm 8 \mathrm{sec}$ & $30 \pm 0 \mathrm{sec}$ & $60 \pm 0 \mathrm{sec}$ & $37 \pm 0 \mathrm{~min}$ & $20 \pm 0 \mathrm{~min}$ & $62 \pm 0 \mathrm{~min}$ \\
\hline
\end{tabular}

Note: Reported times refer to the complete evaporation of solvent. In most cases, glycine crystal appears before the complete evaporation of the solvent. Morphology of "as prepared" glycine crystals was determined from optical images using a minimum of 100 crystal count. Significant differences in the shapes of glycine polymorphs allows for visual determination to be carried out.

their hydrophilic nature) than HMA-and UDET-modified SIFs that are more hydrophobic. It is thought that the hydrophilic/hydrophobic nature of SIFs [9] will have significant effect on the crystallization of glycine. The more hydrophilic MUDA-modified SIFs and moderately hydrophilic HMA-modified SIFs afford for a quicker solvent evaporation time due to faster heat transfer to SIFs and more dispersed glycine crystals. The use of hydrophobic UDET-modified SIFs reduces the surface area of the initial glycine solution exposed to, hence the time for complete evaporation of solvent is increased and glycine crystals are grown on a smaller surface.

Table 2 summarizes the crystallization time and morphology of glycine crystals grown from 3.2 M glycine solutions at $\mathrm{pH} 4,6$,and 9 on HMA-, MUDA- and UDETmodified SIFs at room temperature and microwave heating (results for glass slides not shown to avoid repetition of previously published data). The morphology of the glycine crystals was determined by observing a minimum of $\sim 100$ crystals throughout the surface of SIFs using optical microscope images and SEM images (Fig. 1 and Fig. S1, Supporting Information). It is important to note that we did not attempt to quantify the purity of the glycine crystals from optical images due to the semiquantitative nature of this technique. Fig. 1 also shows that the size of the glycine crystals grown on all surface engineered SIFs using microwave heating visually appears larger than those grown on the identical surfaces at room temperature. The size range for glycine crystals grown on all engineered SIFs will be discussed later in the text in conjunction with the effect of volume of initial glycine solution.

At $\mathrm{pH} 4$, the glycine molecule exists as a positively charged ion due to the protonated amine group $\left(-\mathrm{NH}_{3}{ }^{+}\right)$. Since the isoelectric point of glycine is at about $\mathrm{pH} 6$, it is a zwitterion and at $\mathrm{pH}>6$, its carboxylic group is deprotonated $\left(-\mathrm{COO}^{-}\right)$. At room temperature (i.e. no
Table 3. The effect of sample volume on the length of glycine crystals grown from $3.2 \mathrm{M}, \mathrm{pH}=6$ initial solution at room temperature (RT) and using MA-MAEC technique.

\begin{tabular}{|c|c|c|c|c|c|c|}
\hline & \multicolumn{3}{|c|}{$\begin{array}{c}\text { Length of crystals / } \mu \mathrm{m} \\
\text { Microwave Heating }\end{array}$} & \multicolumn{3}{c|}{$\begin{array}{c}\text { Length of crystals / } \mu \mathrm{m} \\
\text { Room Temperature }\end{array}$} \\
\hline $\begin{array}{c}\text { Volume/ } \\
\text { mL }\end{array}$ & HMA & MUDA & UDET & HMA & MUDA & UDET \\
\hline $\mathbf{5}$ & $90-322$ & $93-800$ & $72-553$ & $68-174$ & $62-389$ & $63-500$ \\
\hline $\mathbf{1 0}$ & $92-558$ & $97-957$ & $80-512$ & $48-288$ & $40-358$ & $51-612$ \\
\hline $\mathbf{2 0}$ & $110-710$ & $101-1020$ & $116-788$ & $52-400$ & $90-350$ & $70-785$ \\
\hline $\mathbf{5 0}$ & $177-1010$ & $112-1200$ & $152-1020$ & $119-450$ & $120-354$ & $81-1250$ \\
\hline $\mathbf{1 0 0}$ & $64-950$ & $110-574$ & N/A & $100-510$ & $112-450$ & $162-1650$ \\
\hline
\end{tabular}

microwave heating), for a fixed volume of $20 \mu \mathrm{L}$ solution of glycine at $\mathrm{pH} 4,6$ and 9, the total crystallization time (i.e., complete evaporation of solvent) on MUDAmodified SIFs was 21, 17 and 20 min, respectively. Glycine crystals grown on MUDA-modified SIFs were mostly $\alpha$-form (Fig. 1). The total evaporation time was drastically reduced with the introduction of microwave heating (i.e., MA-MAEC): 26-35 sec, which corresponds to a 30- to 48-fold decrease in crystallization time as compared to room temperature crystallization. Microwave heating also afforded for the increase in the purity of the glycine crystals: $\sim 100 \%$ pure $\alpha$-glycine crystals were grown from initial glycine solution at $\mathrm{pH}=6$, which provided the best results for MA-MAEC-based crystallization of glycine in terms of production time and purity. Microwave heating of glycine solution at $\mathrm{pH}=4$ and 9 did not yield any discernible glycine crystals on surface engineered SIFs.

At room temperature and on moderately hydrophilic surfaces of HMA-modified SIFs, the total crystallization times were longer than that observed from MUDAmodified SIFs: 15, 26 and $37 \mathrm{~min}$ for initial glycine solution of $\mathrm{pH} 4,6$, and 9, respectively. Glycine crystals grown on HMA-modified SIFs were mostly $\alpha$ - and $\gamma$-form (Fig. 1). The introduction of microwave heating of glycine solutions on HMA-modified SIFs resulted in complete evaporation of the solvent in 30-68 sec, which corresponds to a 30- to 34-fold decrease in crystallization time as compared to room temperature crystallization. On HMA-modified SIFs, $\sim 100 \%$ pure $\alpha$-glycine crystals were grown from initial glycine solution at $\mathrm{pH}=6$ similar to MUDA-modified SIFs. The use of UDETmodified SIFs for the crystallization of glycine at room temperatureresulted in the growth of glycine crystals from initial glycine solution at $\mathrm{pH}=4$ and 6 , which was completed in 38 and $34 \mathrm{~min}$, respectively. No discernible glycine crystals were observed at room temperature when the $\mathrm{pH}$ of the initial glycine solution was increased to 9 . Although the solvent evaporated at quicker times (60$132 \mathrm{sec}$ ) using microwave heating, no glycine crystals were grown on UDET-modified SIFs. This observation can be attributed to the faster evaporation of solvent in combination with the lack of nucleation sites for glycine crystals. From these results one can deduce that the crystallization of glycine using MA-MAEC technique is more effective on MUDA- and HMA-modified SIFs. 


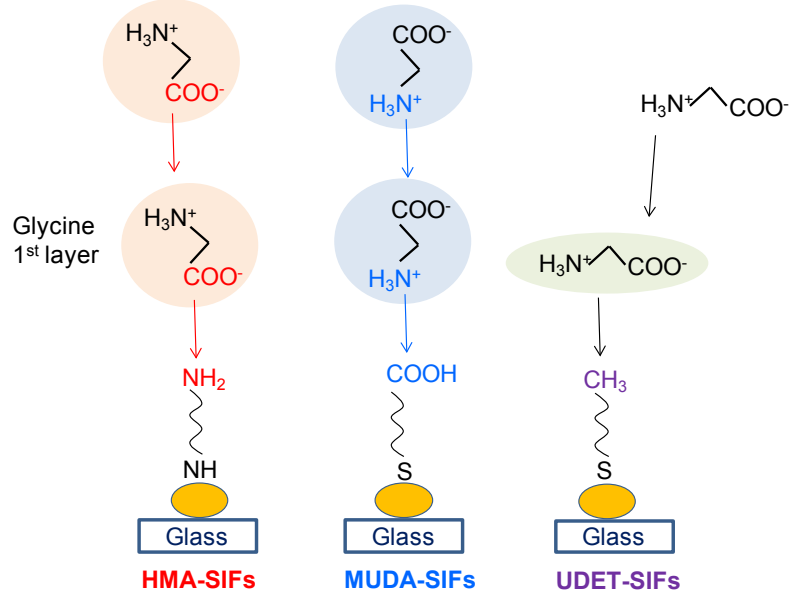

Scheme 1. Schematic depiction of proposed attachment mechanisms of glycine to surface engineered SIFs

As it was described in our previous publications [79], the growth of amino acid crystals occur during the evaporation of the solvent and the complete evaporation of the solvent is not required in MA-MAEC-based crystallization. Since amino acid crystals nucleate, attach and grow on surfaced engineered SIFs during MAMAEC-based crystallization, one can harvest amino acid crystals by removing the solvent at any time. In this study, the solvent is completely evaporated to investigate the full effect of solvent evaporation. In order to demonstrate that glycine crystals are indeed grown during microwave heating, optical images of the initial solution on surface engineered SIFs were intermittently taken by stopping the microwave heating process and capturing the microscope image within $\sim 5-10 \mathrm{sec}$ each time. These images are shown in Fig. S2 (Supporting Information). Glycine crystals were observed after 10 seconds of microwave heating of initial glycine solution on MUDA-modified SIFs, while the appearance of glycine crystals takes a minimum of 5 minutes at room temperature. It took 40 seconds for the complete evaporation of solvent using MA-MAEC technique without significant visual changes to the glycine crystals form 20 to 40 seconds.

In our publications on MA-MAEC-based crystallization of amino acids to date [7-9], the volume of the initial amino acid solution was set to $20 \mu \mathrm{L}$. In this work, the effect of the volume of initial glycine solution on the growth of glycine crystals was also investigated. In this regard, initial glycine solution volumes up to $20 \mu \mathrm{L}(5,10$ and $20 \mu \mathrm{L}$ ) were used without modification to the surface engineered SIFs deposited onto glass slides (dimensions: Length $\mathrm{x}$ width $=75 \times 25 \mathrm{~mm}^{2}$ ). For volumes greater than $20 \mu \mathrm{L}$, commercially available silicone isolator with a single well were used to accommodate the initial glycine solution on surface engineered SIFs. Subsequently, 5, $10,20,50$ and $100 \mu \mathrm{L}$ (largest possible volume for the silicone isolator used) of $\mathrm{pH} 6$ glycine solution was pipetted onto the surface engineered SIFs and the total time taken for complete evaporation of solvent at room temperature and with microwave heating was recorded and is shown in Fig. 2. At room temperature, there was a slight increase in the total time for complete evaporation of solvent on MUDA-modified SIFs as the volume of the initial glycine solution was increased. The increase in total time was more pronounced on UDET- and HMAmodified SIFs. For UDET-modified SIFs, the total evaporation time increased from 19 minutes for $5 \mu \mathrm{L}$ of initial glycine solution to 70 minutes for $100 \mu \mathrm{L}$ of initial glycine solution. In MA-MAEC-based crystallization experiments, the total evaporation time was constant for
A

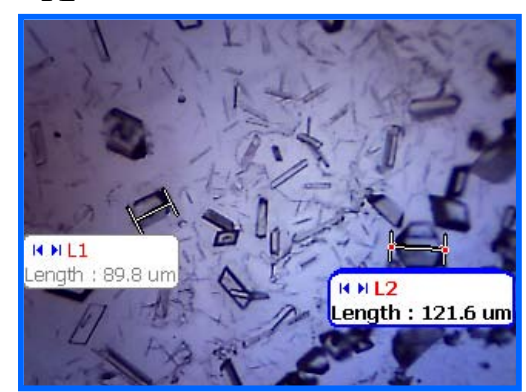

D

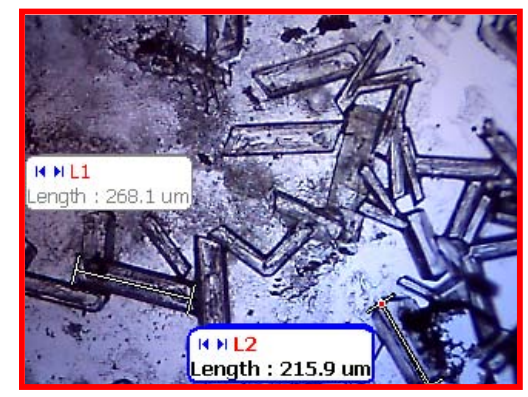

B

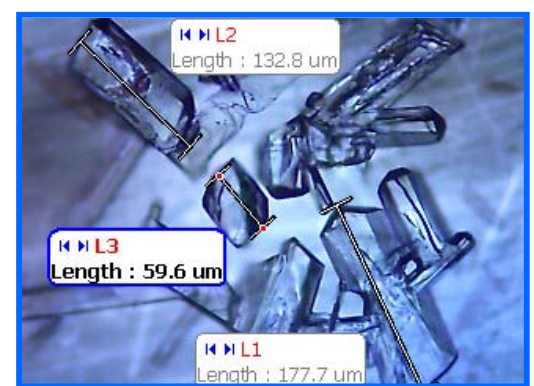

E

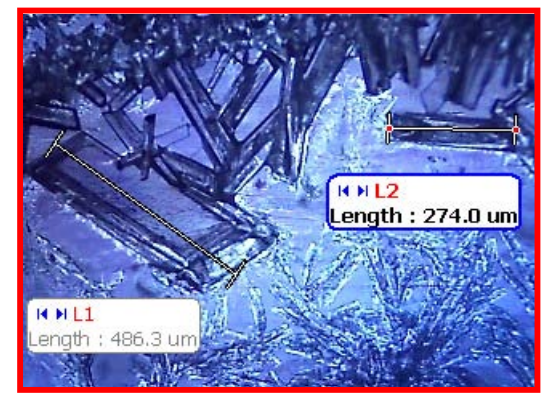

C

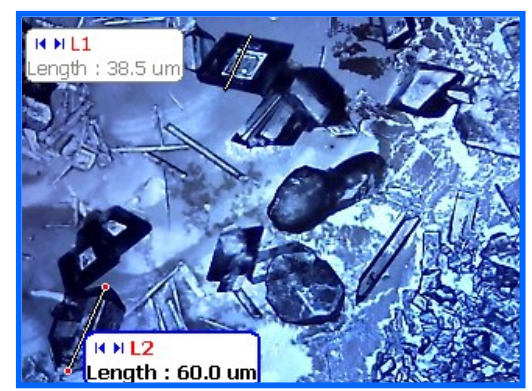

F

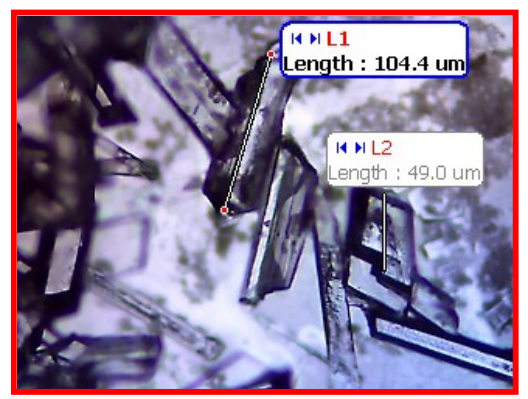

Fig. 1. Optical images of glycine crystals grown from 3.2 M solution at pH 6 on HMA-, MUDA-, and UDET-modified SIFs at room temperature (A, B and C, respectively) and using MA-MAEC technique (D, E and F, respectively). 

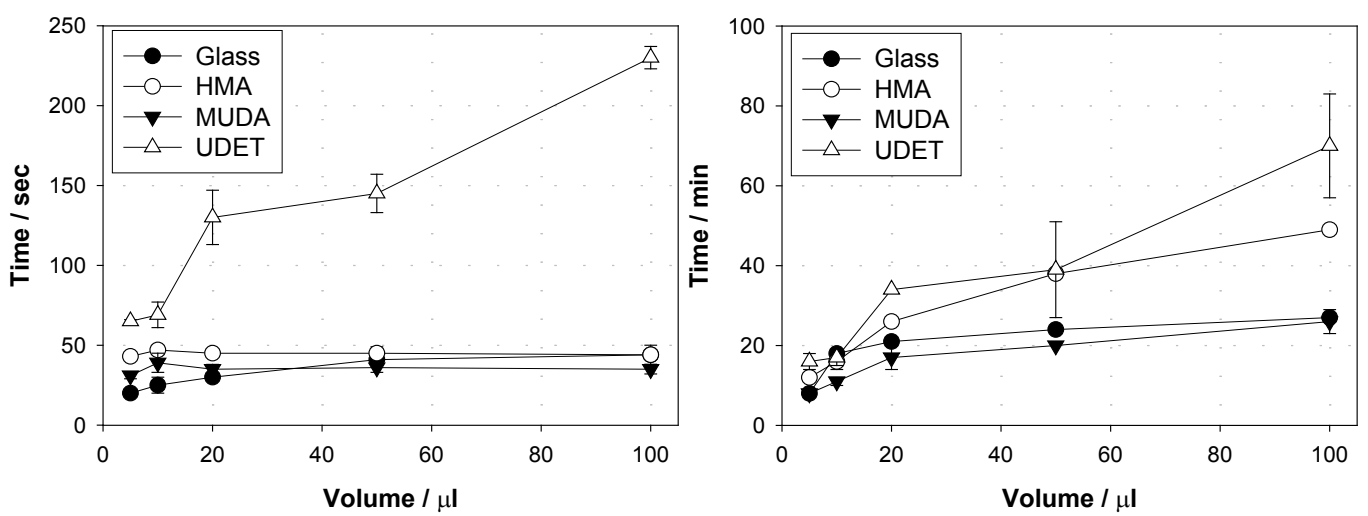

Fig. 2. The effect of sample volume on the total evaporation time for glycine crystals grown from $3.2 \mathrm{M}$, $\mathrm{pH}=6$ initial solution at room temperature (RT) and using MA-MAEC technique on glass and surface engineered surfaces.

"As Prepared", Microwave Heating $3.2 \mathrm{M}, \mathrm{pH} \mathrm{6,20 \mu \textrm {L }}$

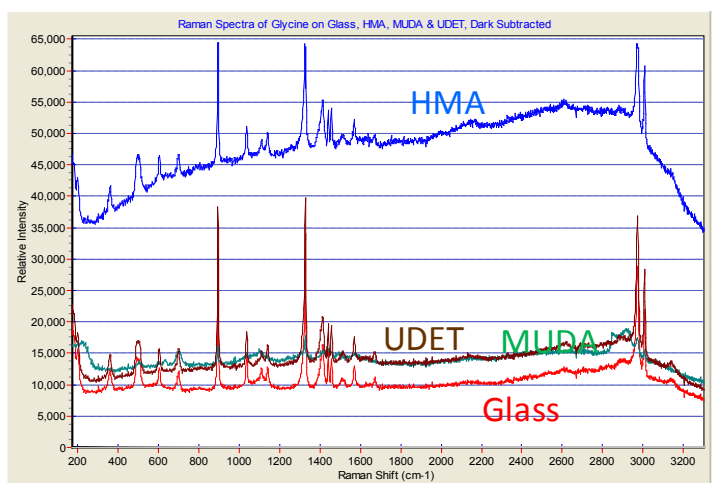

"As Prepared", Room Temperature $3.2 \mathrm{M}, \mathrm{pH} \mathrm{6,} 20 \mu \mathrm{L}$

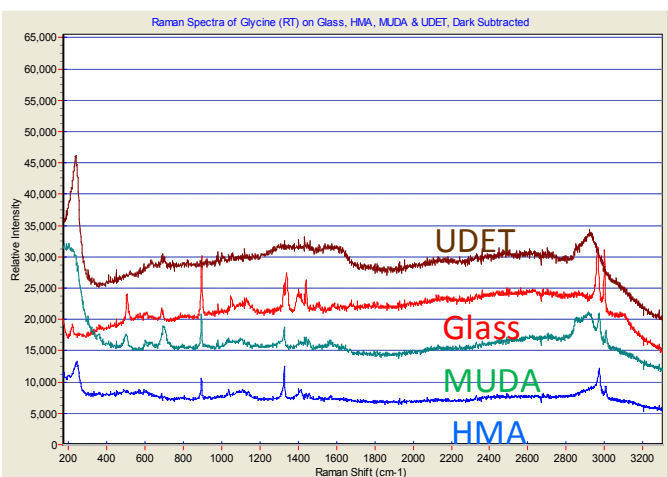

Fig. 3. Raman spectrum of glycine crystals grown on blank glass slides and engineered surfaces at room temperature and using MA-MAEC technique. "As Prepared" refers to glycine crystals attached to the surfaces.

/home/kadir/Desktop/XRD/IMG files/muda32mw6_0001.img: 2-theta Scan

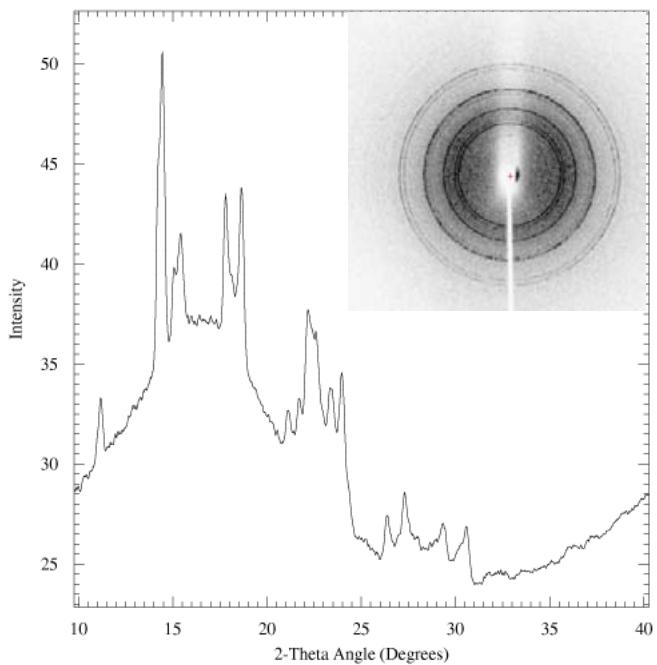

/home/kadir/Desktop/XRD/IMG files/muda32rt6_0001.img: 2-theta Scan

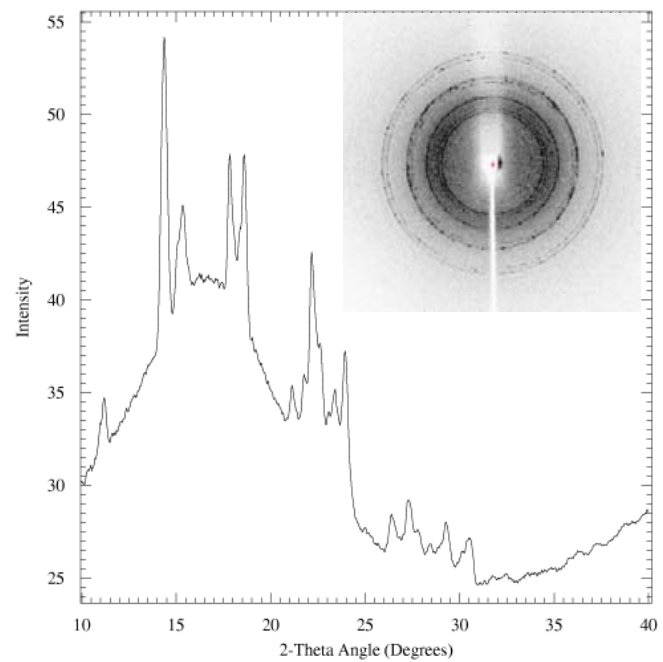

Fig. 4. Powder XRD of glycine crystals grown on MUDA-modifed SIFs at room temperature and using MA-MAEC technique. "Powdered Crystals" refers to glycine crystals harvested from the surfaces and crushed to form powder. 
HMA- and MUDA-modified SIFs. On UDET-modified SIFs, the total evaporation time increased from 60 seconds for $5 \mu \mathrm{L}$ to 235 seconds for $100 \mu \mathrm{L}$ of initial glycine solution. In addition to the total evaporation times, the size of the glycine crystals grown from initial glycine solution with different volumes was investigated and summarized in Table 3. In almost all cases, the increase in the volume of initial glycine solution resulted in an increase the range of glycine crystal size. At room temperature, glycine crystals of $\sim 1.65 \mathrm{~mm}$ size (grown from $100 \mu \mathrm{L}$ of initial glycine solution) were observed on UDET-modified SIFs, which corresponds to a $\sim 3$-fold increase in size as compared to glycine crystals grown from $5 \mu \mathrm{L}$ of initial glycine solution. In MA-MAECbased crystallization experiments, the size range of glycine crystals was also increased as the volume of initial glycinesolution was increased up to $50 \mu \mathrm{L}$ (decreased for $100 \mu \mathrm{L}$ samples). The growth of larger glycine crystals from larger volume solution can be attributed to the availability of larger amount of glycine molecules for crystal growth.

Characterization of glycine crystals grown on surface engineered SIFS was done using Raman spectroscopy and powder XRD. Fig. 3 shows the Raman spectra of glycine crystals on surface engineered SIFs at room temperature and with microwave heating. The Raman peaks obtained from glycine crystals on the surface engineered SIFs were comparable with those grown on blank glass slides (control experiment) room temperature and microwave heating. This observation proves that the glycine crystals grown on surface engineered SIFs and microwave heating were identical to those grownusing conventional technique (blank glass slides and room temperature). The crystals were also characterized using powder XRD. As shown in Fig. 4 and Fig. S3-S6 (Supporting Information), the diffraction peaks obtained from glycine crystals on MUDA-modified SIFs at room temperature and microwave heating were identical. This observation provides further evidence that the MAMAEC technique produces glycine crystals, which are comparable with other methods. Hence, one can grow glycine crystals using the MA-MAEC technique and surface engineered SIFs in a fraction of the time of the conventional evaporative crystallization technique without compromising the glycine crystal structure. Aslan Research Group is currently working on the development of a new crystallization platform designed for microwave heating, where one can crystallize 21 different samples "on demand" and all at once. The findings of these efforts to increase the capacity of crystal production will be published in due course.

\section{Conclusions}

The optimization of MA-MAEC technique for rapid crystallization of glycine by investigating the effect of surface functionality through engineered SIFs as well as the sample volumeis reported. The functionalization of SIFs affected the solvent evaporation time and crystal morphology. Using MUDA- and HMA-modified SIFs, the total evaporation time was decreased to 35 and 45 seconds, respectively. UDET-modified SIFs did not result in growth of glycine crystals using the MA-MAEC technique. This study demonstrated that hydrophilic MUDA- and HMA-modified SIFs are favored for the growth of glycine crystals using the MA-MAEC technique. Sample volume (in the range of 5-100 $\mu \mathrm{L}$ ) did not affect the total crystallization time but resulted in increase in the size of the glycine crystals. Raman and powder XRD data showed that glycine crystals grown on surface engineered SIFs using MA-MAEC technique were identical to those grown using conventional evaporative crystallization technique.

\section{Acknowledgements}

The project described was supported by Award Number 5-K25EB007565-05 from the National Institute of Biomedical Imaging and Bioengineering. The content is solely the responsibility of the authors and does not necessarily represent the official views of the National Institute of Biomedical Imaging and Bioengineering or the National Institutes of Health.

\section{References}

1. Garetz, B.A., J. Matic, and A.S. Myerson, Polarization switching of crystal structure in the nonphotochemical light-induced nucleation of supersaturated aqueous glycine solutions. Phys Rev Lett, 2002. 89(17): p.175501. http://dx.doi.org/10.1103/PhysRevLett.89.175501

2. Hamilton, B.D., et al., Manipulating Crystal Orientation in Nanoscale Cylindrical Pores by Stereochemical Inhibition. J. Am. Chem. Soc.,2009. 131(7): p. 2588-2596. http://dx.doi.org/10.1021/ ja807193s

3. Lee, A.Y., et al., Crystallization on confined engineered surfaces: A method to control crystal size and generate different polymorphs. $J$. Am. Chem. Soc., 2005. 127(43): p. 14982-14983. http://dx.doi.org/ $10.1021 / \mathrm{ja} 055416 \mathrm{x}$

4. Singh, A. and A.S. Myerson, Chiral self assembled monolayers as resolving auxiliaries in the crystallization of valine.J. Pharm. Sci., 2010. 99(9): p. 3931-40.

5. Lee, I.S., A.Y. Lee, and A.S. Myerson, Concomitant polymorphism in confinedenvironment.Pharm Res, 2008. 25(4): p. 960-8. http:// dx.doi.org/10.1007/s11095-007-9424-z

6. Trout, B.L., et al., Surface Design for Controlled Crystallization: The Role of Surface Chemistry and Nanoscale Pores in Heterogeneous Nucleation.Langmuir, 2011. 27(9): p. 5324-5334. http://dx.doi. org/10.1021/la104351k

7. Pinard, M.A. and K. Aslan, Metal-Assisted and MicrowaveAccelerated Evaporative Crystallization.Cryst Growth Des, 2010. 10 (11): p. 4706-4709. http://dx.doi.org/10.1021/cg101059c

8. Pinard, M.A., et al., Rapid crystallization of 1 -arginine acetate on engineered surfaces using metal-assisted and microwaveaccelerated evaporative crystallization. Cryst Eng Comm, 2012. http://dx.doi.org/10.1039/c2ce25380a

9. Alabanza, A.M., E. Pozharski, and K. Aslan, Rapid Crystallization of L-Alanine on Engineered Surfaces using Metal-Assisted and Microwave-Accelerated Evaporative Crystallization.Cryst Growth Des, 2012. 12(1): p. 346-353. http://dx.doi.org/10.1021/cg2011502

10. Mohammed, M., et al., Rapid and Selective Crystallization of Acetaminophen using Metal-Assisted and Microwave-Accelerated Evaporative Crystallization.Nano Biomed Eng, 2012. 4(1): p. 35-40.

11. Abel, B., et al., Plasmon-Enhanced Enzymatic Reactions: A Study of Nanoparticle-Enzyme Distance- and Nanoparticle Loading Dependent Enzymatic Activity.Nano Biomed Eng, 2011. 3(3): p. 184-191.

Copyright:(c) 2012 T.A.J. Grell, et al. This is an open-access article distributed under the terms of the Creative Commons Attribution License, which permits unrestricted use, distribution, and reproduction in any medium, provided the original author and source are credited. 